
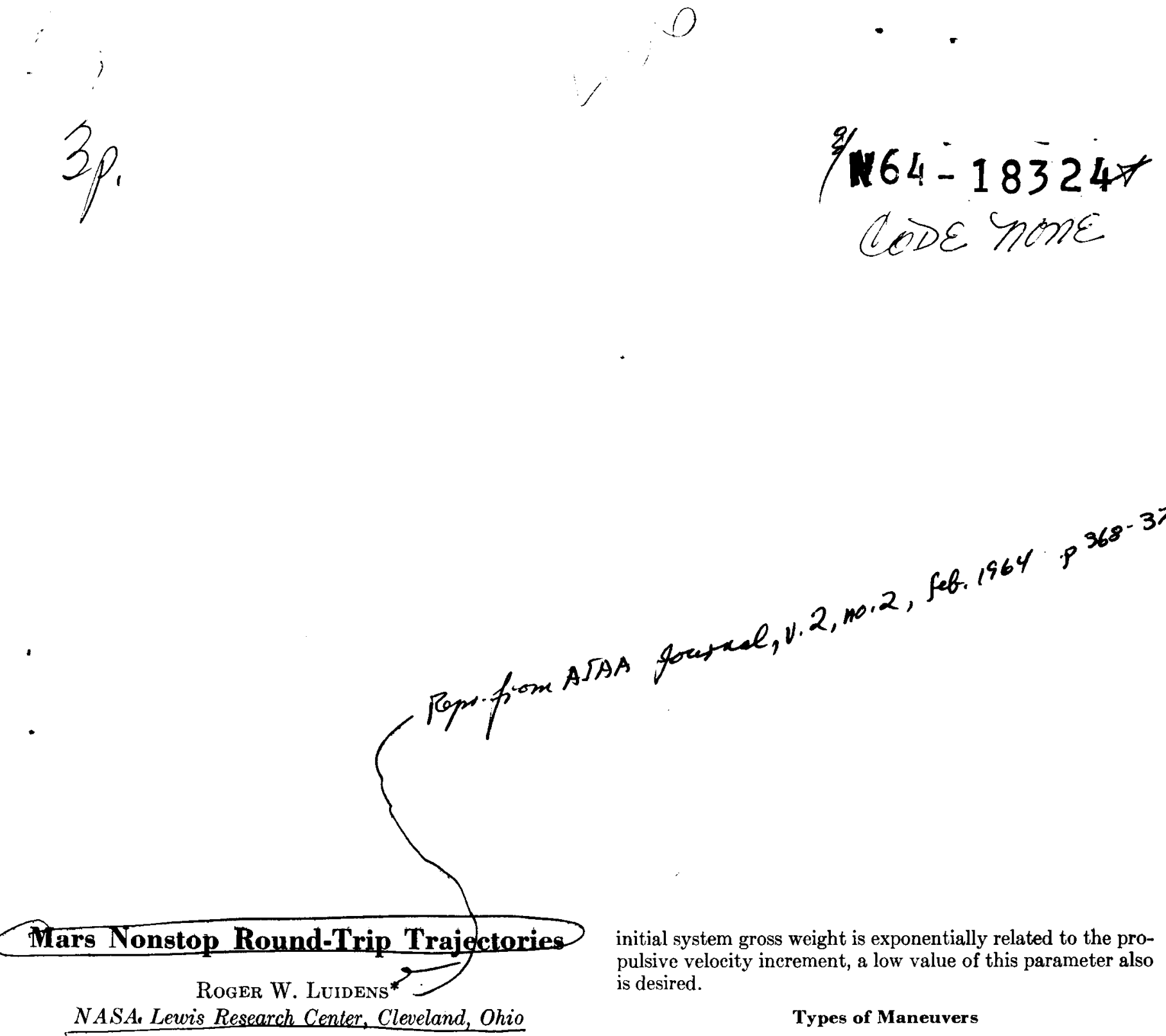

\title{
Introduction
}

$\mathbf{M}$ ANNED and unmanned nonstop round trips may be precursors of the first manned landing on Mars. The vehicle for such a trip will be launched from Earth and, without stopping, fly by Mars and return to Earth. Except in special cases, a vehicle placed on a trajectory to Mars will not return to Earth. In many cases, however, an Earth return can be achieved if the trajectory is modified by one of the following means: 1) by the gravity of Mars, 2) by gravity supplemented by propulsion, or 3) by gravity supplemented by aerodynamic forces. The analysis of the trajectories resulting from the latter two trajectory modifications is the contribution made herein.

In this note, the preceding three kinds of nonstop roundtrip trajectories are compared in the years 1971 and 1980 on the basis of mission time and the required propulsive velocity increment. A short mission is desirable for reducing life support system weight and for psychological reasons. Since the

Received October 17, 1963. The author wishes to acknowledge the assistance of Jay M. Kappraff and Richard J. Flaherty in conducting the present analysis.

* Head, Flight Systems Section, Mission Analysis Branch. Associate Fellow Member AIAA.

Copyright, 1964, by the American Institute of Aeronautics and Astronautics, and reprinted by permission of the copyright owner

initial system gross weight is exponentially related to the propulsive velocity increment, a low value of this parameter also is desired.

\section{Types of Maneuvers}

The trajectory of a typical nonstop round-trip mission in 1971 is superimposed on orbits of Mars and Earth in Fig. 1. Note that the Mars orbit is quite eccentric. The vehicle leaves Earth at point 1, passes close to Mars at point 2, and returns to Earth at point 3 . In general, outbound and inbound legs of the trajectory are of unequal length.

When the vehicle passes Mars, its trajectory can be changed in three ways, which characterize the three trajectories analyzed (Fig. 2). For a gravity turn, only the gravitational field of Mars deflects the vehicle (Fig. 2a). The arrival and the departure velocities $V_{A}$ and $V_{D}$ are equal in magnitude, and the turning is equally distributed between the arrival and the departure phases of the maneuver, that is, $\Phi_{G A}=\Phi_{G D}$.

If gravity alone cannot produce an Earth return trajectory, the trajectory can be further changed by thrusting. The thrust is generally best applied at the sphere of influence when the vehicle departs from Mars. Figure 2a illustrates a velocity increment $\Delta V_{\sigma^{\prime}}$ imparted to change the departure velocity vector from $V_{D}$ to $V_{D^{\prime}}$. A trajectory using this maneuver is called a propulsive-gravity turn.

An acrodynamic-turn trajectory (Fig. 2b) may be used to advantage when a low departure velocity and a high turning angle are required. The total turning can be broken into three stages. First, the arrival velocity vector is turned through an angle $\Phi_{G A}$ by the planetary gravitation field. Second, after entering the Mars atmosphere, the vehicle 


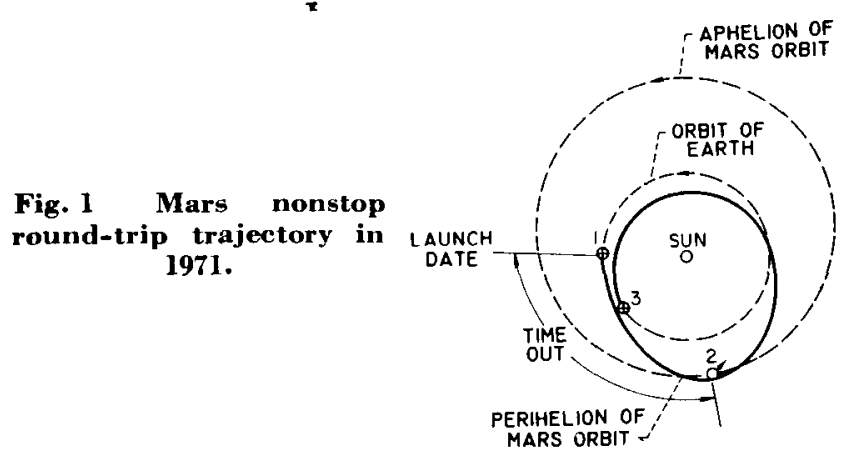

velocity vector is turned through an angle $\Phi_{\alpha}$ by the vehicle lift and is also decreased in magnitude because of aerodynamic drag. Finally, after the vehicle leaves the Mars atmosphere and until it departs from the sphere of influence of Mars, it is turned through an angle $\Phi_{G D}$.

\section{Method of Analysis}

The round-trip trajectories were studied by first selecting the type of nonstop trajectory, the launch year, and the total trip time (equivalent to the central angle from 1 to 3 in Fig. 1). Then, by varying the launch date and the time out, the trajectories with a minimum propulsive velocity increment were located. The trajectory data were generated by the "patch-conic, impulsive $\Delta V$ " computer program of Ref. 1 , which accounts for both the eccentricity and the inclinations of the Earth and the Mars orbits.

\section{Results and Discussion}

The minimum propulsive velocity increment for each mission time, $\Delta V$, in miles per second is plotted against the mission time in days for launch years of 1971 and 1980 in Figs. 3 and 4 , respectively. In all cases, the mission starts from an Earth parking orbit of 1.1 Earth radii, and atmospheric braking is used at Earth return. The lowest periapsis of the Mars pass considered for the gravity and the propulsive-gravity-turn trajectories is 1.1 Mars radii. For references, the total $\Delta V$ required for a comparable direct lunar landing and return mission $(5.3 \mathrm{mps})$ and the minimum $\Delta V$ for a one-way fly-by transfer to Mars (about $2.2 \mathrm{mps}$ ) are indicated on the ordinate.

Many of the Mars round trips require a $\Delta V$ substantially less than that for the lunar landing. The Mars trip times, however, are considerably longer than the lunar trip time, which is of the order of a week.

Gravity-turn trajectories are possible only at total trip times of approximately 1 year and again at trip times greater than about $1 \frac{1}{2}$ years. If 1980 is the launch year, the lowest $\Delta V$ for a gravity turn occurs for a trip time of 540 days.

By admitting the use of thrusting near Mars, the propulsivegravity-turn type of trajectory, round trips become possible
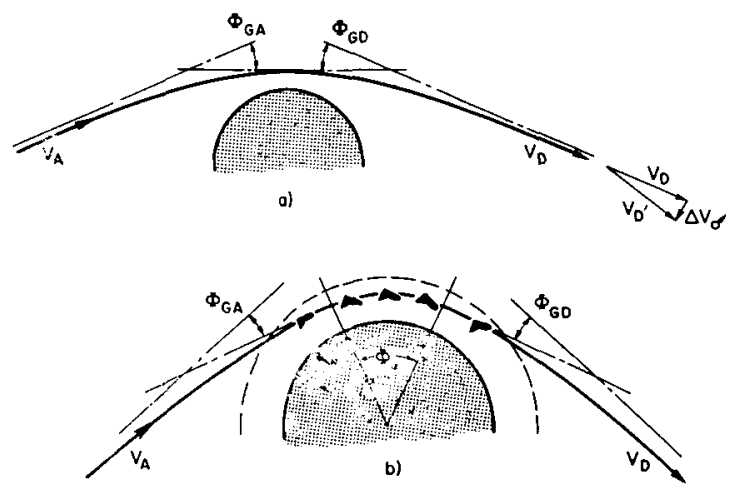

Fig. 2 Types of nonstop round-trip trajectories: a) gravity turn and propulsive-gravity turn; b) atmospheric

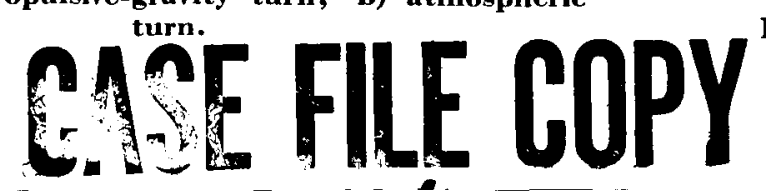

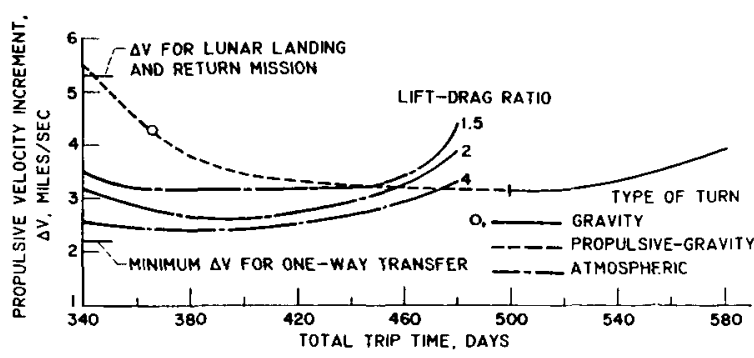

Fig. 3 Round-trip nonstop missions in 1971.

over a wide range of trip times. For 1980, the minimum $\Delta V$ is slightly less than that for the best gravity turn and occurs at a shorter trip time, about 500 days. Also, in both 1971 and 1980 , there is only a slow increase in $\Delta V$ with decreasing trip time for trip times down to about 400 days.

Even further reductions in $\Delta V$ occur when an aerodynamic turn is used at Mars. Values only slightly greater than that for a one-way transfer occur for a vehicle with a lift-drag ratio of 4 . Significant reductions in $\Delta V$ below that for the propulsive-gravity turn exist also for the more practical lift-drag values of 1.5 and 2.0. The trip times for low $\Delta V$ are between about 380 and 420 days for this case.

The effect of launch year on the required $\Delta V$ may be seen by comparing the values in Figs. 3 and 4 . For the gravity and the propulsive-gravity turns, the values of $\Delta V$ are greater in 1980 than in 1971. This effect is due to the eccentricity of the Mars orbit. The trips in 1971 pass Mars when Mars is near its perihelion, as illustrated in Fig. 1. The trips in 1980 , however, pass Mars when Mars is near its aphelion. A comparison of the atmospheric turns in 1971 and 1980 shows that the required propulsive velocity increment for this type of trajectory is least sensitive to the launch year.

Several factors not discussed in this note are also important in the case of atmospheric turns: $g$ loads, heat loads, and feasible lift-drag ratios. In most cases, the $g$ loads during the atmospheric turns are less than 10 Earth $g$ 's, which is about the limit of human tolerance. The vehicle must be capable of absorbing both the heat load associated with the aerodynamic turn at Mars and the Earth atmospheric entry. The lowest $\Delta V$ available will depend on the practically realizable liftdrag ratios, which may be of the order of 2.0. The heat loads also depend on the vehicle lift-drag ratio. The atmosphericturn trajectories pose challenging but not unfeasible aerodynamic and heat-protection problems, which require further study.

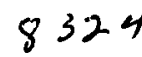

Conclusions

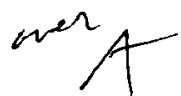

The following conclusions can be drawn from the present study of Mars nonstop round-trip trajectories using gravity, propulsive-gravity, and atmospheric turns at Mars:

1) When compared with gravity turns, the use of small amounts of propulsion near Mars markedly expands the range

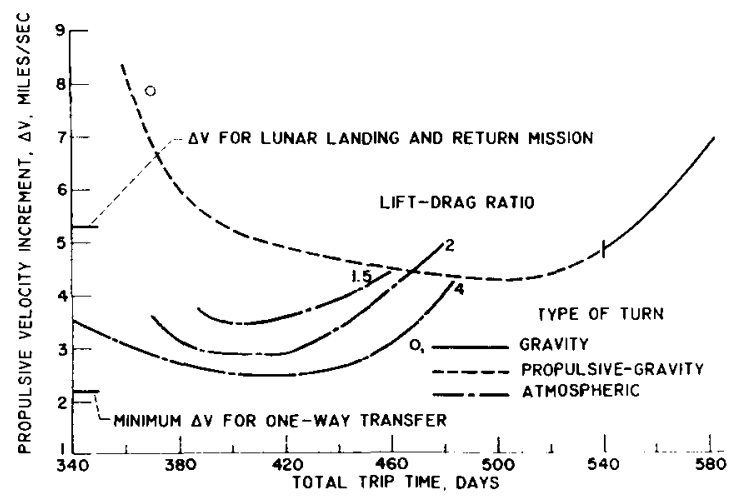

Fig. 4 Round-trip nonstop missions in 1980. 
of possible trip times and in some cases reduc the required propulsive velocity increment.

2) The use of an atmospheric turn at Mar can reduce the required propulsive velocity increment to a value approaching that for a one-way transfer to Mars, depending on the feasible vehicle lift-drag ratios. Low propulsive velocity increments occur for trip times down to about 1 year.

3) In general, greater propulsive velocity increments are required in 1980 than in 1971 . The propulsive velocity in-

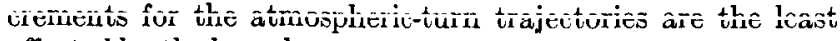
affected by the launch year.

4) The propulsive-gravity and the atmospheric-turn trajectories appear to offer the potential of fast, light-weight, nonstop round trips to Mars.

\section{Reference}

${ }^{1}$ Knip, G., Jr. and Zola, C. L., "Three-dimensional trajectory analysis for round-trip missions to Mars," NASA TN D-1316 (1962). 